Published in final edited form as:

J Am Chem Soc. 2016 March 23; 138(11): 3627-3630. doi:10.1021/jacs.5b11876.

\title{
Conformational Rearrangements of Individual Nucleotides during RNA-Ligand Binding Are Rate-Differentiated
}

\author{
Marina Frener and Ronald Micura* \\ Institute of Organic Chemistry and Center for Molecular Biosciences, University of Innsbruck, \\ 6020 Innsbruck, Austria
}

\begin{abstract}
A pronounced rate differentiation has been found for conformational rearrangements of individual nucleobases that occur during ligand recognition of the pre $\mathrm{Q}_{1}$ class-I riboswitch aptamer from Thermoanaerobacter tengcongensis. Rate measurements rely on the 2ApFold approach by analyzing the fluorescence response of riboswitch variants, each with a single, strategically positioned 2-aminopurine nucleobase substitution. Observed rate discrimination between the fastest and the slowest conformational adaption is 22-fold, with the largest rate observed for the rearrangement of a nucleoside directly at the binding site and the smallest rate observed for the $3^{\prime}$ unpaired nucleoside that stacks onto the pseudo-knot-closing Watson-Crick base pair. Our findings provide novel insights into how compact, prefolded RNAs that follow the induced-fit recognition mechanism adapt local structural elements in response to ligand binding on a rather broad time scale and how this process culminates in a structural signal that is responsible for efficient downregulation of ribosomal translation.
\end{abstract}

\begin{abstract}
Assessment of kinetics for ligand-induced structure rearrangements encountered in RNA riboswitches is crucial to comprehend the intricate mechanism of these RNA domains that regulate gene expression.1 Riboswitches are usually located within the 5' noncoding regions of bacterial mRNA and consist of an aptamer and an adjoining expression platform. 2 They bind metabolites with high selectivity and specificity to mediate control of transcription or translation. In a manner that is dependent on metabolite concentration, nascent mRNAs containing riboswitch domains can enter one of two mutually exclusive folding pathways to impart regulatory control; the outcomes of these folding pathways correspond to ligandbound (LB) or -free (LF) states. Thereby, the aptamer folds trigger structural cues into the expression platform which, in turn, transduces a "on" or "off" signal for gene expression.
\end{abstract}

One of the most critical steps in riboswitch gene regulation is ligand-sensing by the aptamer. This process, in particular the induced conformational changes at the secondary and tertiary structure level, can be thoroughly analyzed in vitro by the 2 ApFold approach.1d The approach relies on synthetic RNAs that correspond to a transcriptional folding intermediate. These RNAs usually comprise the riboswitch aptamer down to a defined transcriptional

\footnotetext{
*Corresponding Author ronald.micura@uibk.ac.at. Notes

The authors declare no competing financial interest.
} 
pause site.2b,3 Furthermore, the individual RNAs contain a single 2-aminopurine (Ap) that is a fluorescent isomer of adenine. The position for the nucleobase replacement is selected on the basis of the 3D structure of the LB aptamer by following the criteria of minimal structural interference; original H-bonding and base-stacking patterns are to be retained.1d Ideally, the Ap replaces a nucleobase that undergoes a pronounced conformational change in response to ligand binding. Nucleotides that become exposed upon ligand binding of the RNA (e.g., a single nucleotide (nt) bulge) or involved in a tertiary structure formation (e.g., a pseudo-knot) are well-suited. The concomitant change in Ap fluorescense intensity can then be traced spectroscopically and analyzed kinetically.4 We stress that an inherent influence on the rate occurring from the nucleobase replacement itself can be kept minor by following the abovementioned criteria but cannot be completely excluded.

Here we investigate folding of the pre $\mathrm{Q}_{1}$ class-I riboswitch5 from the thermophilic organism Thermoanaerobacter tengcongensis (Tte). This translation-controlling riboswitch consists of an aptamer of only $33 \mathrm{nt}$ 's, with its $3^{\prime}$ end comprising the first nucleosides of the ribosome binding site (Shine-Dalgarno sequence, SD).5m The present understanding is that regulation relies on stabilizing the pseudo-knot fold of preQ $_{1}$-I RNA through ligand binding, making the SD sequence nonaccessible to the ribosome. Previous studies also suggested that the free riboswitch is prefolded and that pseudo-knot formation can occur without ligand in solution. $5 \mathrm{k}, 6$ Indeed, the LF but pseudo-knot-forming aptamer was successfully crystallized and its structure solved by X-ray analysis.5g,m

We used the 2ApFold approach to analyze the ligand-induced structural adaptions of this RNA fold at single-nt resolution. Surprisingly, our investigations revealed the broadest differentiation in rates for rearrangements of this kind thus far reported for riboswitch aptamers. Our findings impact the understanding of RNA/small-molecule interactions that follow an induced-fit (IF) mechanism; 7 the rates we determined are consistent with initial contacts between ligand and pocket under concomitant fast RNA conformational adaptions, followed by slower ones in more distant regions from the binding site.

First, we examined the Watson-Crick (WC) base pair (bp) C9-G33 that padlocks the aptamer fold in the northern sphere (Figure 1). This terminal bp is part of the functionally decisive pseudo-knot and not involved in additional $\mathrm{H}$ bonding networks, but it stacks onto the A10-A32 platform below (Figure 1). For the isosteric U9-Ap33 variant, addition of $\mathrm{Mg}^{2+}$ at physiologically relevant concentrations of $2 \mathrm{mM}$ resulted in a minor decrease of fluorescence, indicating only a small gain in pseudo-knot preformation. However, the fluorescence of Ap33 becomes significantly quenched upon $\mathrm{pre}_{1}$ addition in saturating concentration. This suggests that the $3^{\prime}$-terminal nt becomes fully base-paired only in the presence of ligand that is in accordance with its functional role in SD sequestration. $K_{293 \mathrm{~K}}$ was determined to be $(37.4 \pm 0.11) \times 10^{3} \mathrm{M}^{-1} \mathrm{~s}^{-1}$, under pseudo-first-order conditions and on the basis of single-exponential curve fitting of the fluorescence responses (Figure 2, Table 1, and Supporting Information (SI)).

Compaction in the northern part of the aptamer fold (Figure 1) through ligand binding is not only reflected in bp formation between nt's 33 and 9. Intricate aptamer architecture prompted us to evaluate an Ap variant that senses formation of the A32.A10 platform just 
below it (Figure 1). Fluoresence of the A10Ap variant was clearly decreased when $\mathrm{Mg}^{2+}$ was added, indicating a notable preorganization of the platform in the presence of the divalent ions. Nevertheless, two-thirds of the total fluorescence response was obtained when preQ $_{1}$ ligand addition was executed. Interestingly, $k_{293 \mathrm{~K}}$ for this process was determined to be $(2.37 \pm 0.23) \times 10^{3} \mathrm{M}^{-1} \mathrm{~s}^{-1}, \sim 15$-fold slower compared to 33-9 bp formation in direct neighborhood (Figure 2 and Table 1 ).

Compaction of the northern part of the aptamer fold upon $\mathrm{preQ}_{1}$ binding is also sensed by the unpaired nucleoside 34 subsequent to the closing of bp 33-9. Corresponding G34Ap variant displayed a rather high degree of fluorescence quenching upon $\mathrm{Mg}^{2+}$ addition and additionally upon preQ $_{1}$ addition. Relaxation into the final, stacked conformation is slow, with $k_{293 \mathrm{~K}}=(1.84 \pm 0.01) \times 10^{3} \mathrm{M}^{-1} \mathrm{~s}^{-1}$ (Figure 1 , Table 1 , and SI).

Turning our attention to the southern hemisphere of the aptamer, we substituted U22, which adopts an exposed conformation in the ligand-aptamer complex, by Ap (Figure 1). We observed a significant increase in fluorescence upon pre $\mathrm{Q}_{1}$ addition, nicely projecting the binding process. This nt in position 22 is rather distant from the ligand pocket. $k_{293 \mathrm{~K}}$ was determined to be $(11.27 \pm 0.50) \times 10^{3} \mathrm{M}^{-1} \mathrm{~s}^{-1}$; this value is in the middle range compared with the previously determined ones (Figure 2, Table 1, and SI).

Finally, we set out to trace the conformational rearrangement of a specific nucleoside at the heart of the riboswitch. In the LF crystal structure, A14 mimics the preQ ${ }_{1}$ ligand and spreads into the binding pocket, stacked between A11 and C16 (Figures 1 and 3A).5m However, when preQ $_{1}$ ligand becomes bound, A14 is shifted into a tightly stacked position between C15 and A13, forming a base triplet (trans WC/sugar edge A14-G11) with the preformed G11-C30 WC bp (Figures 1 and 3B).5m At the same time, the nucleobase C15 undergoes a marked rotation from a looped-out position to bp formation with the $\mathrm{WC}$ face of $\mathrm{preQ}_{1}$ (Figure 3A,B). Corresponding to stacked positions of Ap14 in both the free and the LB aptamers, $5 \mathrm{~m}$ the absolute fluorescence intensities were weaker compared to those of the previous examples. Nevertheless, a clear response to $\mathrm{Mg}^{2+}$ and significant fluorescence quenching upon preQ ${ }_{1}$ addition were observed; for the latter, $k_{293}=(41.7 \pm 0.9) \times$ $10^{3} \mathrm{M}^{-1} \mathrm{~s}^{-1}$ was determined. This value stands for the fastest conformational adaption measured for the Tte preQ $_{1}$ riboswitch aptamer (Figure 2 and Table 1).

Together the kinetic data support a folding model in which ligand binding induces the fastest conformational adaptions at the pocket of the prefolded RNA. Further adaptations spread out to peripheral regions, thereby compacting the northern hemisphere and sequestering the first nucleosides of the SD sequence. Slower conformational adaptions are consistent with this scenario. For example, stacking of the terminal unpaired purine on top of the northern hemisphere occurs with the lowest rate measured, 22-fold slower than that of nt adjustment in the core.

We note that the apparent ligand affinities of C9U-G33Ap, A10Ap, and G34Ap RNAs were almost identical: U22Ap and A14Ap RNAs had 2.4-fold lower and 2.6-fold higher affinities, respectively, whereas the range of rates $k_{\text {fold }}$ of all five variants varied 22-fold. Affinities of the Ap variants were additionally measured by an independent method, namely, isothermal 
calorimetry (ITC). Absolute affinity values determined by ITC were slightly larger, but they varied only by a factor of 2.3 (SI). This confirms that the Ap replacements were wellselected and only minimally interfering in the native structure. Further support for the integrity of our folding model stems from a series of RNA controls, as described below.

RNA control ctrl 1 targets bp G11-C30, which is observed in both free and LB X-ray structures of the Tte preQ ${ }_{1}$ aptamer. It represents the "ceiling" of the ligand pocket.5g,m Because this interaction could be a crucial determinant for preorganization of the small aptamer, we were wondering if replacement by U11-A30 is tolerated. In principle, an A-U bp can reintegrate into the original base quadruplet (Figure S1-A in SI) because $\mathrm{H}$ bond networks to $\mathrm{C} 7$ and A14 are largely retained. As analyzed by the corresponding U22Ap variant (Figure S1-B in SI), the G11A-C30U mutations resulted in 4-fold loss of affinity $\left(K_{\mathrm{d} \text {,app }}=218 \mathrm{nmol}\right.$; Table 1 and SI $)$. Ap22 folding kinetics were decreased only 2-fold with $k_{293 \mathrm{~K}}=(4.59 \pm 0.55) \times 10^{3} \mathrm{M}^{-1} \mathrm{~s}^{-1}$ (Table 1). Importantly, Ap fluorescence did not respond to $\mathrm{Mg}^{2+}$ addition (Figure $\mathrm{S} 1-\mathrm{B}$ in $\mathrm{SI}$ ); this observation suggests that $\mathrm{Mg}^{2+}$-induced preorganization of the pocket does not occur or is less pronounced compared to that of the native aptamer. When $\mathrm{C} 7$ was mutated to $\mathrm{U} 7$ in addition to G11A-C30U (crtl 2), thereby replacing the triplet $\mathrm{C} 30-\mathrm{G} 11 \cdot \mathrm{C} \mathrm{H}^{+}$by $\mathrm{U} 30-\mathrm{A} 11 \cdot \mathrm{U} 7$, affinity to $\mathrm{preQ}_{1}$ was drastically reduced by an additional factor of $30\left(K_{\mathrm{d}, \text { app }}=6.7 \mu \mathrm{mol}\right.$; Table 1 , SI $)$.

Using ctrl 3, we investigated the impact of the mutation C15U (Figure S1-C in SI). This mutation directly interferes with ligand recognition by shifting the bp mode with $\mathrm{preQ}_{1}$ from WC to wobble. Again, the mutation was analyzed via the corresponding UAp22 variant. Not unexpectedly, affinity was lowered ( 14-fold, $K_{\mathrm{d} \text {,app }}=0.7 \mu \mathrm{M}$; Table 1$)$, but the Ap22 folding kinetics were increased $\sim 2$-fold $\left(k_{293 \mathrm{~K}}=(20.50 \pm 1.90) \times 10^{3} \mathrm{M}^{-1} \mathrm{~s}^{-1}\right)$. This indicates a faster dynamic exchange of $\mathrm{pre}_{1}$ ligand with the mutated binding pocket compared to the native one, also implying a significantly increased off-rate.

Control RNAs ctrl 4 and ctrl 5 were synthesized to test the influence of the exchange of the pseudoknot-closing bp C9-G33 by U9-A33. For ctrl 4 with Ap14 as fluorescence sensor, $k_{293 \mathrm{~K}}=(45.29 \pm 2.40) \times 10^{3} \mathrm{M}^{-1} \mathrm{~s}^{-1}$, comparable to that of "wt" A14Ap RNA (Table 1). This observation is consistent with the unchanged binding pocket and its close environment (G11-C30 ceiling; G5-C16 "floor") where A14Ap is located and interacting. Interestingly, the C9U-G33A mutation slightly impacts on the conformational adaption of Ap22 (RNA ctrl 5); the rate was 2-fold lower compared to that of "wt" U22Ap RNA.

$\mathrm{PreQ}_{1}$ class-I riboswitches have been investigated extensively over the years. Some selected aspects are discussed in the light of the results obtained here. The Tte $\mathrm{preQ}_{1}$ riboswitch was analyzed in detail by single-molecule FRET spectroscopy.6f Walter et al. found strong evidence that this aptamer recognizes its ligand by an IF mechanism, $6 \mathrm{f}$ which requires a prefolded RNA. The data here now provide insights into the kinetic order of conformational adjustments of selected nucleosides (of the prefolded RNA) in response to ligand binding. The obtained $k_{\text {fold }}$ reveal a 22 -fold discrimination. This is the broadest range observed for riboswitch aptamers so far. It was only 7-fold for thiamine pyrophosphate (TPP), $4 \mathrm{~b} 3$-fold for adenine,4a and 2-fold for the $S$-adenosylmethionine (SAM) class II aptamers.8 Although the kinetic data for this comparison stemmed from a single method (2ApFold) only, we note 
that for one system a comparison with NMR spectroscopically determined single-residue kinetics can be made. For the B. subtilis xpt-pbuX guanine-sensing riboswitch, Schwalbe et al. found two time regimes (for bp formation) in response to ligand-binding:9 A faster time course with $t_{1 / 2}$ in the range 18-23 $\mathrm{s}$ was observed for residues that are directly involved in formation of the ligand-binding pocket; a slower process in the time range of 27-31 s was observed for nts in helices P2 and P3 and in the loop/loop region (L2 and L3) of the RNA. This corresponds to $\sim 1.5$-fold differentiation, comprising the analysis of all aptamer nucleosides. The $2 \mathrm{ApFold}$ approach for the related add adenine riboswitch gave a 3-fold differentiation of rates for conformational adaptions of $\sim 10$ strategically positioned Ap variants and is in good accordance.

Another aspect addressed using NMR methods concerned the rearrangement of A14, for which the fastest rate was measured. This finding can be rationalized because the conformational change occurs at the pocket of the prefolded RNA and is directly associated with ligand binding. Very likely in a concerted movement, $\mathrm{preQ}_{1}$ pushes the placeholder A14 out of the pocket and $\mathrm{C} 15$ concomitantly locks this arrangement by rotating from the looped out position to bp with $\mathrm{preQ}_{1}$ inside. We previously probed preformation of the G11C30 bp ("ceiling") based on a selectively labeled ${ }^{15} \mathrm{~N}(1)-\mathrm{G} 11 /{ }^{15} \mathrm{~N}(3)-\mathrm{C} 30$ pair.10 In the ${ }^{1} \mathrm{H}_{-}{ }^{15} \mathrm{~N}$ HSQC spectra of the free RNA, we observed two resonances for G11 with clear correlations to $\mathrm{C} 30$ in the HNN COSY spectra, corresponding to two major populations of conformers with a preformed pseudo-knot in solution.10 It was speculated that these two conformers in slow exchange may represent the conformer with C15 exposed and A14 stacked as placeholder, whereas the other one may represent the conformer with empty pocket. We note that the structural distinctions between free and bound folds of the Tte aptamer are not necessarily the same as those for preQ $_{1}$ riboswitches from other organisms. For B. subtilis, Feigon et al. showed by NMR that the free $\mathrm{preQ}_{1}$ aptamer forms an extended stem P1 that includes the nucleoside corresponding to Tte A14 in a WC bp; it is clearly distinct from the Tte free fold.5d

Noteworthy, ligand binding kinetics of the Tte pre $_{1}$ aptamer were analyzed by surface plasmon resonance (SPR) and reported to be $7.77 \times 10^{4} \mathrm{M}^{-1} \mathrm{~s}^{-1} .5 \mathrm{~m}$ The order of this value is consistent with the rates we measured for conformational adaptions at the pocket. In response to a reviewer's request, we analyzed $k_{\text {fold }}$ for a different ligand, 7-cyano-7deazaguanine $\left(\mathrm{preQ}_{0}\right)$, and obtained a rate for the $\mathrm{A} 14 \mathrm{Ap}$ variant that was 12 -fold lower compared to the native ligand (SI). This difference was consistent with the SPRobtained 12fold reduction of the on-rate and again supported the integrity of $2 \mathrm{ApFold}$ for kinetic analysis of single-nt conformational adaptions in RNA. Moreover, binding of $\mathrm{preQ}_{0}$ indicates that $\mathrm{pre}_{1}$ riboswitches possess some elasticity of their pockets that may enable binding of alternative ligand structures. This feature makes riboswitches potential targets in the search for novel compounds with antibacterial activity.2,11 Kinetic studies such as the one presented can contribute to thriving innovative ligand design that is based on the optimization of $k_{\text {fold/unfold }}$ and $k_{\mathrm{on} / \mathrm{off}}$ rates rather than binding affinities $K_{\mathrm{d}}$ alone.

2-Aminopurine fluorescence spectroscopy unveiled the broad rate distribution for conformational adaptions of nucleobase interactions that are induced through ligand binding in the Tte $\mathrm{preQ}_{1}$ riboswitch. Although the biological reasons for the broad distribution 
remain unclear, our findings from ensemble-averaged relaxation kinetics contribute to a better understanding of ligandinduced RNA folding processes on the basic research level. Our data support the induced-fit recognition mechanism for this aptamer by demonstrating that the adaptions spread out from the binding pocket to more distant regions in response to ligand binding. These mechanistic insights together with the finding that the 3D architecture of the RNA complex supports strong pairing of a terminal single bp in the WC mode and propagates to a tightly $3^{\prime}$-stacked unpaired purine provides clues as to how the sequestration of only the first two nucleotides of the SD sequence results in downregulation of ribosomal translation $(\mathrm{SI})$.

\section{Supporting Information}

Refer to Web version on PubMed Central for supplementary material.

\section{Acknowledgments}

Funding by the Austrian Science Foundation FWF (P27947, I1040) is acknowledged.

\section{References}

(1). (a) Al-Hashimi HM, Walter NG. Curr Opin Struct Biol. 2008; 18:321. [PubMed: 18547802] (b) Mustoe AM, Brooks CL, Al-Hashimi HM. Annu Rev Biochem. 2014; 83:441. [PubMed: 24606137] (c) St-Pierre P, McCluskey K, Shaw E, Penedo JC, Lafontaine DA. Biochim Biophys Acta Gene Regul Mech. 2014; 1839:1005.(d) Haller A, Soulière MF, Micura R. Acc Chem Res. 2011; 44:1339. [PubMed: 21678902]

(2). (a) Breaker RR. Mol Cell. 2011; 43:867. [PubMed: 21925376] (b) Garst AD, Edwards AL, Batey RT. Cold Spring Harb Perspect Biol. 2011; 3:a003533. [PubMed: 20943759] (c) Deigan KE, Ferré-D’Amaré AR. Acc Chem Res. 2011; 44:1329. [PubMed: 21615107] (d) Serganov A, Patel DJ. Curr Opin Struct Biol. 2012; 22:279. [PubMed: 22579413] (e) Schwalbe H, Buck J, Furtig B, Noeske J, Wöhnert J. Angew Chem Int Ed. 2007; 46:1212.(f) Serganov A. RNA Biol. 2010; 7:98. [PubMed: 20061809]

(3). Wong TN, Pan T. Methods Enzymol. 2009; 468:167. [PubMed: 20946770]

(4). (a) Rieder R, Lang K, Graber D, Micura R. ChemBioChem. 2007; 8:896. [PubMed: 17440909] (b) Lang K, Rieder R, Micura R. Nucleic Acids Res. 2007; 35:5370. [PubMed: 17693433] (c) Soulière MF, Haller A, Rieder R, Micura R. J Am Chem Soc. 2011; 133:16161. [PubMed: 21882876]

(5). (a) Roth A, et al. Nat Struct Mol Biol. 2007; 14:308. [PubMed: 17384645] (b) McCown PJ, Liang JJ, Weinberg Z, Breaker RR. Chem Biol. 2014; 21:880. [PubMed: 25036777] (c) Klein DJ, Edwards TE, Ferré-D’Amaré AR. Nat Struct Mol Biol. 2009; 16:343. [PubMed: 19234468] (d) Kang M, Peterson R, Feigon J. Mol Cell. 2009; 33:784. [PubMed: 19285444] (e) Spitale RC, Torelli AT, Krucinska J, Bandarian V, Wedekind JE. J Biol Chem. 2009; 284:11012. [PubMed: 19261617] (f) Rieder U, Lang K, Kreutz C, Polacek N, Micura R. ChemBioChem. 2009; 10:1141. [PubMed: 19382115] (g) Liberman JA, Wedekind JE. WIREsRNA. 2012; 3:369.(h) Rieder U, Kreutz C, Micura R. Proc Natl Acad Sci USA. 2010; 107:10804. [PubMed: 20534493] (i) Feng J, Walter NG, Brooks CL III. J Am Chem Soc. 2011; 133:4196. [PubMed: 21375305] (j) Zhang Q, Kang M, Peterson RD, Feigon J. J Am Chem Soc. 2011; 133:5190. [PubMed: 21410253] (k) Petrone PM, Dewhurst J, Tommasi R, Whitehead L, Pomerantz AK. J Mol Graphics Modell. 2011; 30:179.(1) Eichhorn CD, Feng J, Suddala KC, Walter NG, Brooks CL, Al-Hashimi HM. Nucleic Acids Res. 2012; 40:1345. [PubMed: 22009676] (m) Jenkins JL, Krucinska J, McCarty RM, Bandarian V, Wedekind JE. J Biol Chem. 2011; 286:24626. [PubMed: 21592962]

(6). (a) Santner T, Rieder U, Kreutz C, Micura R. J Am Chem Soc. 2012; 134:11928. [PubMed: 22775200] (b) Banas P, Sklenovsky P, Wedekind JE, Sponer J, Otyepka M. J Phys Chem B. 
2012; 116:12721. [PubMed: 22998634] (c) Suddala KC, Rinaldi AJ, Feng J, Mustoe AM, Eichhorn CD, Liberman JA, Wedekind JA, Al-Hashimi HM, Brooks CL III, Walter NG. Nucleic Acids Res. 2013; 41:10462. [PubMed: 24003028] (d) Gong Z, Zhao Y, Chen C, Duan Y, Xiao Y. PLoS One. 2014; 9:e92247. [PubMed: 24663240] (e) Moschen T, Wunderlich CH, Spitzer R, Levic J, Micura R, Tollinger M, Kreutz C. Angew Chem Int Ed. 2015; 54:560.(f) Suddala KC, Wang J, Hou Q, Walter NG. J Am Chem Soc. 2015; 137:14075. [PubMed: 26471732]

(7). (a) Leulliot N, Varani G. Biochemistry. 2001; 40:7947-7956. [PubMed: 11434763] (b) Boehr DD, Nussinov R, Wright PE. Nat Chem Biol. 2009; 25:789. [PubMed: 19841628]

(8). Haller A, Rieder U, Aigner M, Blanchard SC, Micura R. Nat Chem Biol. 2011; 7:393. [PubMed: 21532598]

(9). Buck J, Fürtig B, Noeske J, Wöhnert J, Schwalbe H. Proc Natl Acad Sci USA. 2007; 104:15699_ 15704. [PubMed: 17895388]

(10). Neuner S, Santner T, Kreutz C, Micura R. Chem-Eur J. 2015; 21:11634. [PubMed: 26237536]

(11). Howe JA, et al. Nature. 2015; 526:672. [PubMed: 26416753]

(12). Leontis NB, Westhof E. RNA. 2001; 7:499. [PubMed: 11345429] 


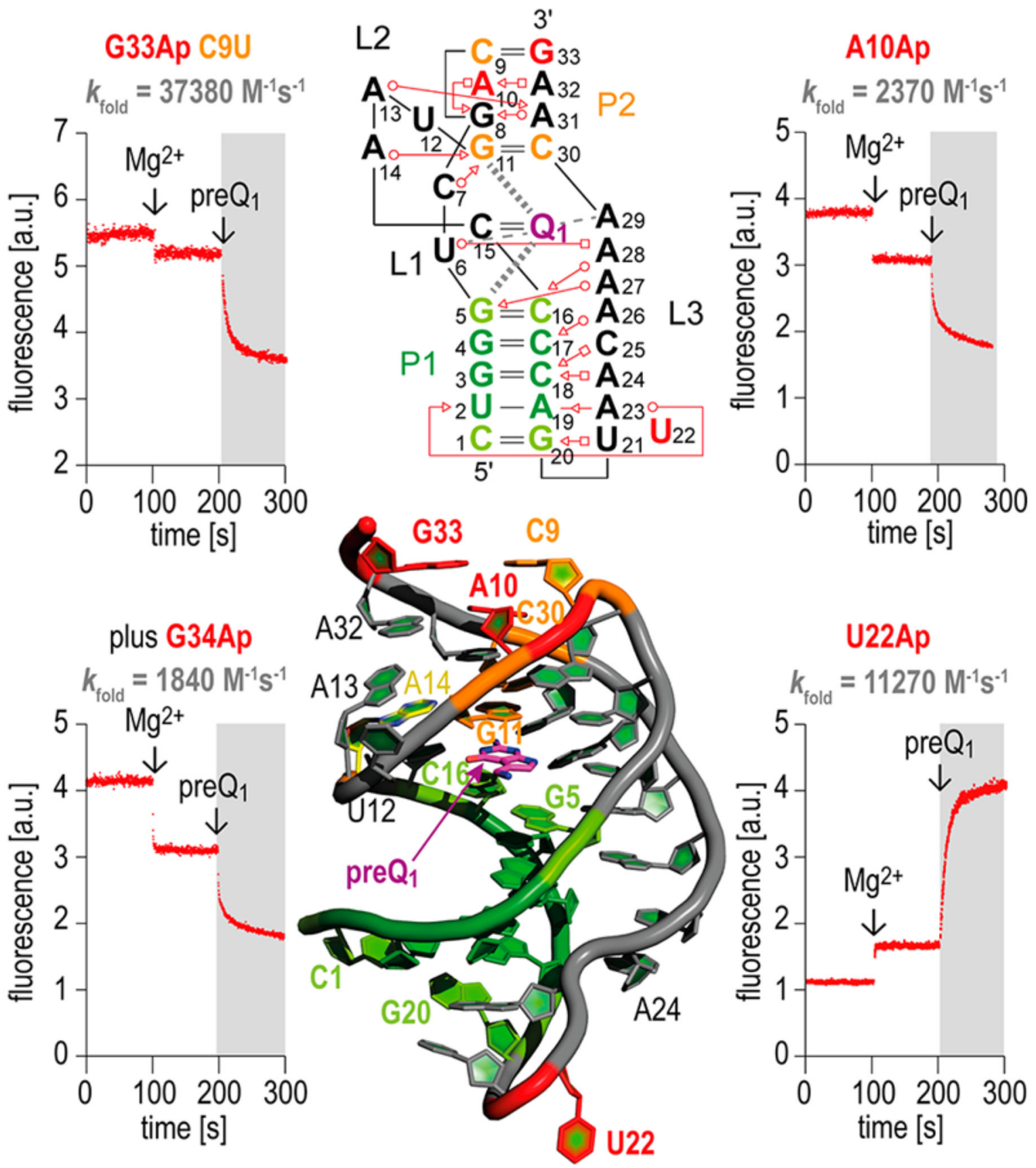

Figure 1.

Ligand-induced conformational rearrangements of the Tte $\mathrm{preQ}_{1}$ riboswitch analyzed by 2aminopurine (Ap) fluorescence. Secondary structure in Leontis-Westhof nomenclature (top middle) 12 and 3D structure presentation (bottom middle, PDB ID: 3Q50).5m Fluorescence traces of four Ap-labeled preQ ${ }_{1}$ RNAs in response to $\mathrm{Mg}^{2+}$ and preQ $_{1}$ ligands. Conditions: $0.5 \mu \mathrm{M}$ RNA, $100 \mathrm{mM} \mathrm{KCl}, 50 \mathrm{mM}$ MOPS, pH 7.5, $293 \mathrm{~K}$. Ligands: $2 \mathrm{mM} \mathrm{MgCl}_{2}, 5 \mu \mathrm{M}$ $\mathrm{preQ}_{1}$. 

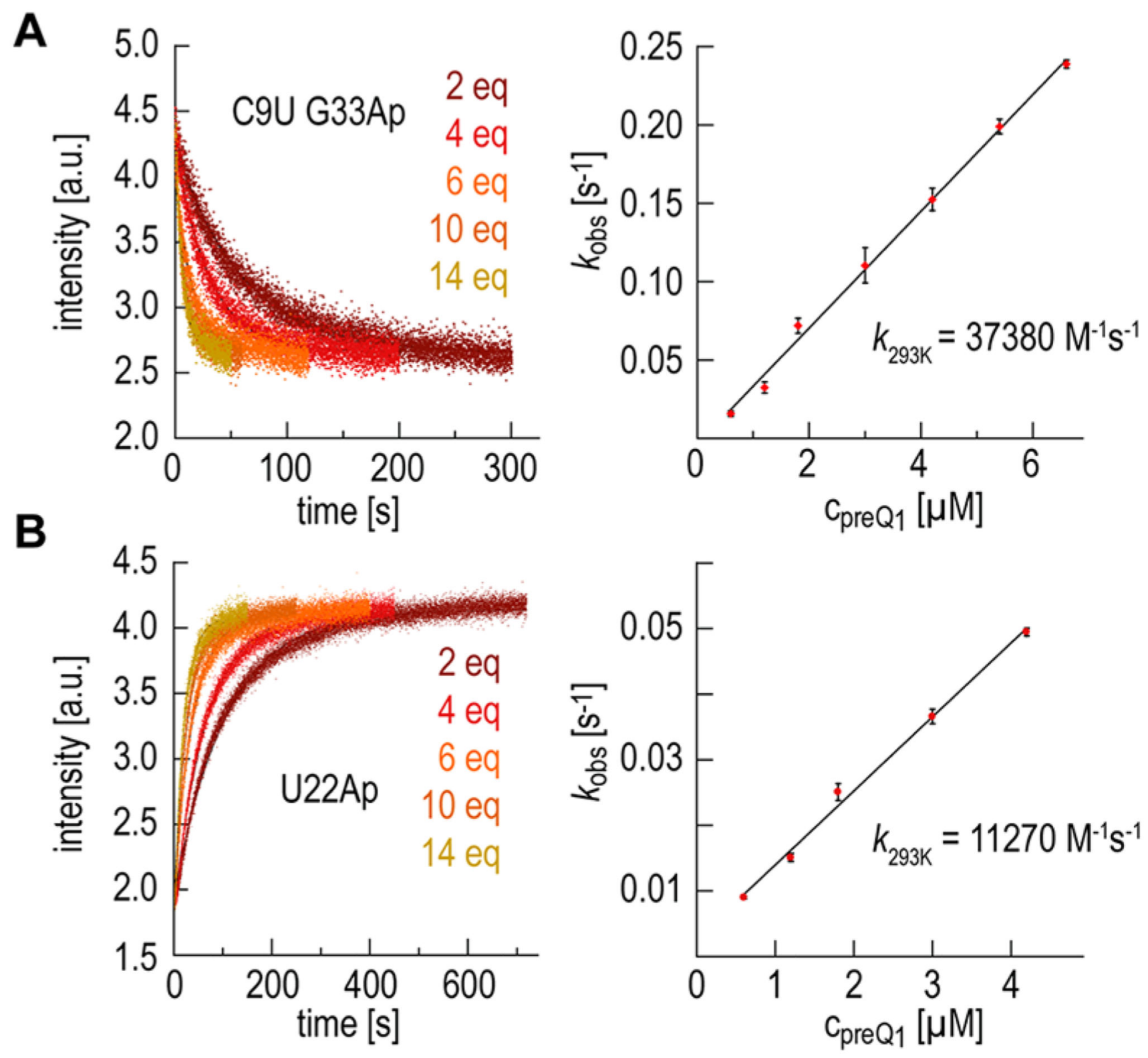

Figure 2.

Stopped-flow fluorescence spectroscopy to monitor the kinetics of nucleobase rearrangements upon preQ $_{1}$ binding. (A) Ap fluorescence traces of the Tte G33Ap C9U variant at different preQ $_{1}$ concentrations (left) (For single-exponential curve fits, see SI.) Rate constant $k_{293}$ from plots of observed rate $k_{\text {obs }}$ vs ligand concentration (right). (B) Same as Abut for the Tte U22Ap variant. $0.3 \mu \mathrm{MRNA}, 2 \mathrm{mM} \mathrm{MgCl}_{2}, 100 \mathrm{mM} \mathrm{KCl}, 50 \mathrm{mM}$ MOPS, pH 7.5, $293 \mathrm{~K}$, preQ $_{1}$ as indicated. 


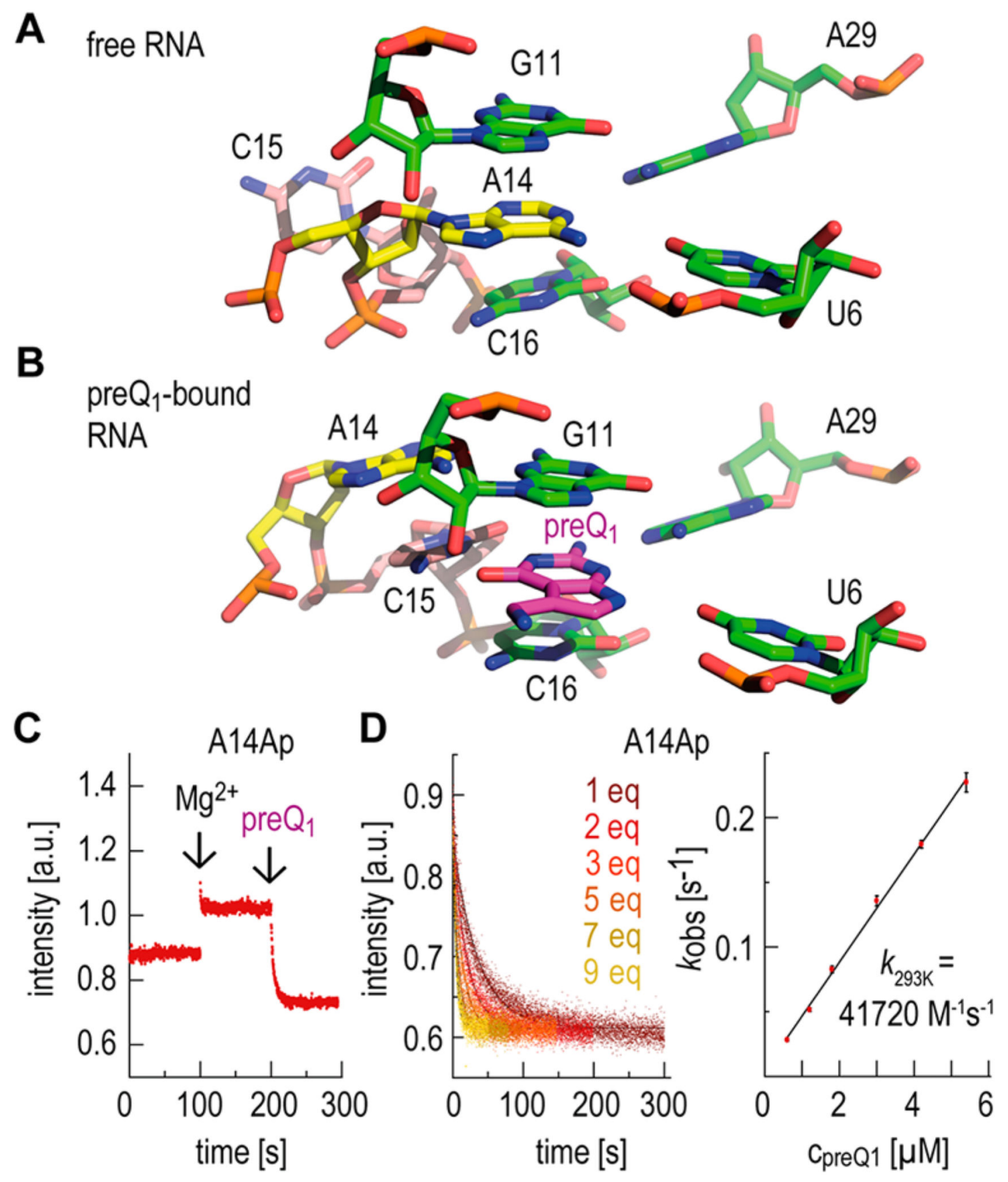

Figure 3.

Structure and kinetics analysis of an A14Ap-modified Tte $\mathrm{preQ}_{1}$ riboswitch aptamer. Binding pockets of (A) free (PDB ID: 3Q51) and (B) preQ ${ }_{1}$-bound RNA (PDB ID: 3Q50). $5 \mathrm{~m}$ A14 replaces the ligand in the free RNA; A14 together with C15 are differently positioned when ligand is bound. (C) Qualitative fluorescence response of the A14Ap variant upon successive addition of saturation concentrations of $\mathrm{Mg}^{2+}(2 \mathrm{mM})$ and $\mathrm{preQ}_{1}$ ligand ( $5 \mu \mathrm{M}$; 10-fold excess over RNA). (D) Stopped-flow fluorescence spectroscopy was used to monitor the kinetics of the Ap14 conformation change upon preQ $\mathrm{Q}_{1}$ binding (left). Ap 
fluorescence traces for different $\mathrm{pre}_{1}$ concentrations. (For single-exponential curve fits, see SI.) Rate constant $k_{293 \mathrm{~K}}$ from plot of observed rate $k_{\mathrm{obs}}$ vs ligand concentration (right). Conditions: $0.6 \mu \mathrm{M}$ RNA, $2 \mathrm{mM} \mathrm{MgCl} 2,100 \mathrm{mM} \mathrm{KCl}, 50 \mathrm{mM}$ MOPS, pH 7.5, $293 \mathrm{~K}$, preQ 1 as indicated. 
Table 1

Rate Constants $\boldsymbol{k}_{\text {fold }}$ and Apparent Binding Constants $K_{\mathrm{D}, \text { app }}$ of Tte PreQ Riboswitch $_{1}$ Variants Determined by Ap Fluoresence ${ }^{a}$

\begin{tabular}{lcc}
\hline \multicolumn{1}{c}{ Ap variant } & $\boldsymbol{k}_{\text {fold,293K }}\left(\mathbf{M}^{-\mathbf{1}} \mathbf{s}^{\mathbf{- 1}}\right)$ & $\boldsymbol{K}_{\mathbf{d , a p p}, \mathbf{2 9 3 K}} \boldsymbol{b}(\mathbf{n M})$ \\
\hline C9U G33Ap & $37380 \pm 1070$ & $21 \pm 4$ \\
A10Ap & $2370 \pm 230$ & $21 \pm 3$ \\
G34Ap & $1840 \pm 10$ & $19 \pm 4$ \\
U22Ap & $11270 \pm 500$ & $51 \pm 5$ \\
A14Ap & $41720 \pm 955$ & $8 \pm 3$ \\
C30U G11A U22Ap (ctrl 1) & $4590 \pm 550$ & $218 \pm 20$ \\
C30UG11A C7U U22Ap (ctrl 2) & n.d. & $6700 \pm 1080$ \\
C15U U22Ap (ctrl 3) & $20500 \pm 1900$ & $710 \pm 50$ \\
C9U G33AU14Ap (ctrl 4) & $45290 \pm 2400$ & $4 \pm 2$ \\
C9U G33A U22Ap (ctrl 5) & $5130 \pm 950$ & $25 \pm 4$ \\
\hline
\end{tabular}

${ }^{a}$ For extended data set, see SI.

${ }^{b}$ For comparison with isothermal titration calorimetry(ITC)-determined $K_{\mathrm{d}}$ values, see SI and main text. 